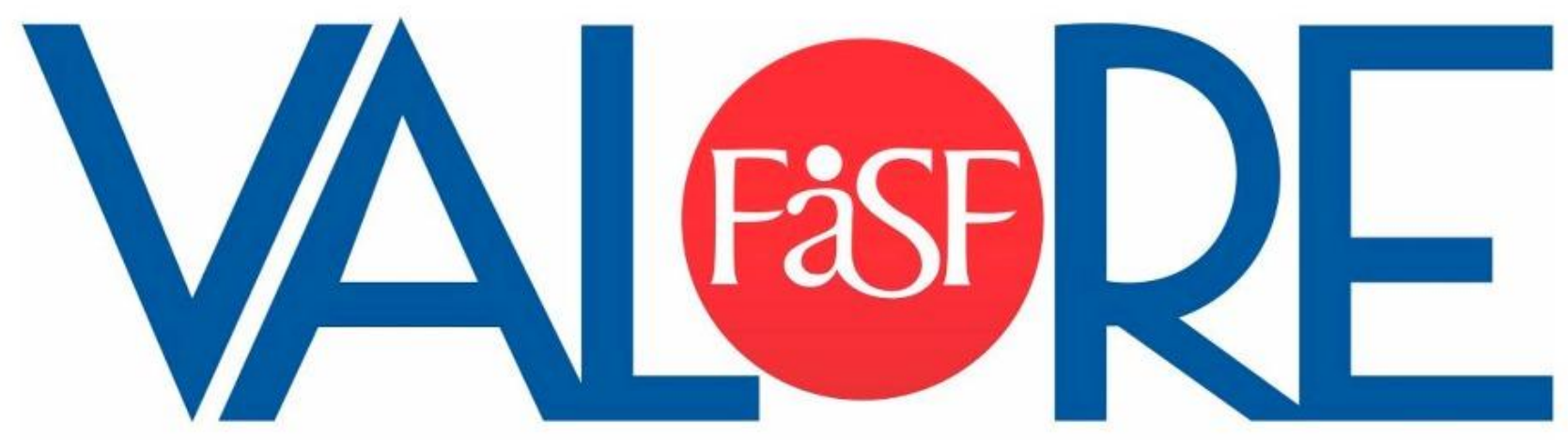

Revista Científica da FASF Faculdade Sul Fluminense

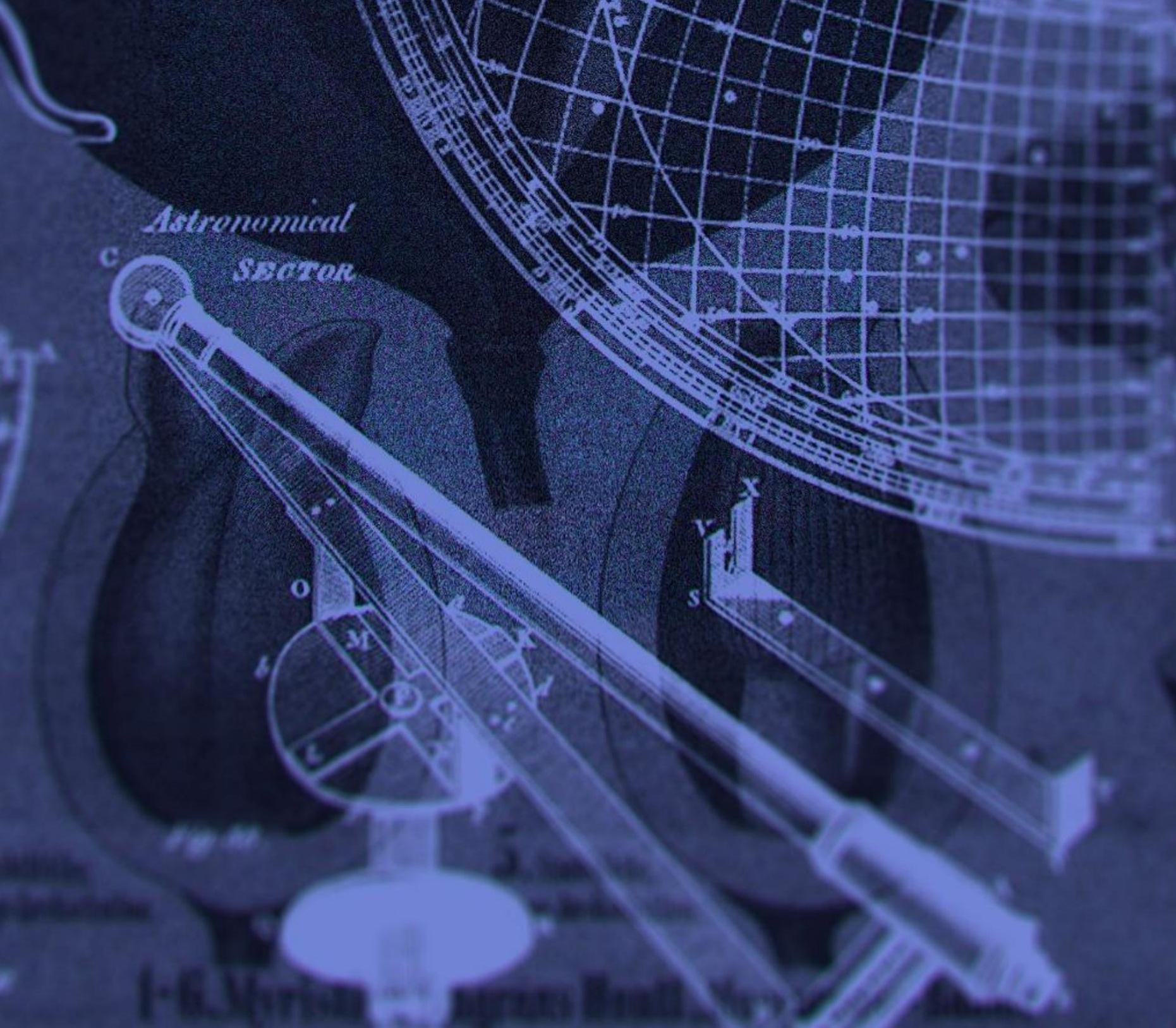




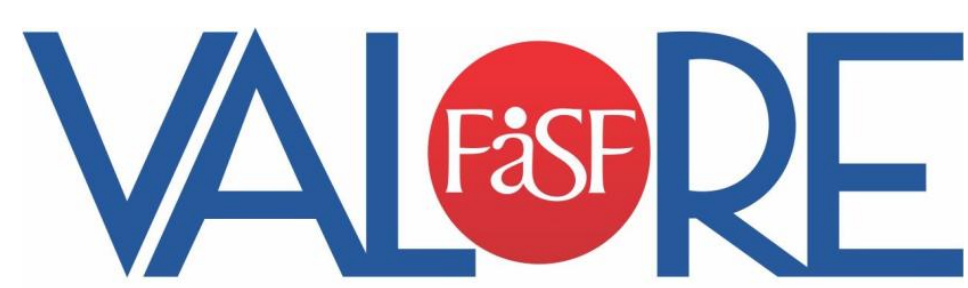

Revista Científica da FASF Faculdade Sul Fluminense 
Revista Valore - Revista Científica da FaSF - Faculdade Sul Fluminense, Volta Redonda, v.2 n.2, 157p. Jul./Dez. 2017

A Revista Valore, criada em junho de 2016, órgão oficial de divulgação da Faculdade Sul Fluminense, com periodicidade semestral, tem a finalidade de divulgar a produção científica das diferentes áreas do saber que seja de interesse das áreas da saúde, ciências humanas, sociais e tecnológicas.

Ficha Catalográfica

Revista Valore. - v.2 n.2(jul/dez/2017) - Volta Redonda: Faculdade Sul Fluminense, Coordenação de Cursos de Graduação e Pós-Graduação da FASF

Revista Semestral Interdisciplinar das Coordenações de Cursos de Graduação e Pós-Graduação da FaSF Descrição Baseada em n.1, 2017.

1 - Periódico Interdisciplinar I. Coordenação de Cursos de Graduação e Pós-Graduação da FaSF. II. Faculdade Sul Fluminense.

Publicada em dezembro de 2017.

Site: https://revistavalore.emnuvens.com.br/valore

Revista Valore - Revista Científica da Faculdade Sul Fluminense.

Endereço: Rua Alberto Cunha Rodrigues, n. ${ }^{\circ} 39$ - Bairro Jardim Amália II - Volta Redonda/RJ

CEP: 27251-220

Telefone: 24-33408771

e-mail: revistavalore@gmail.com 


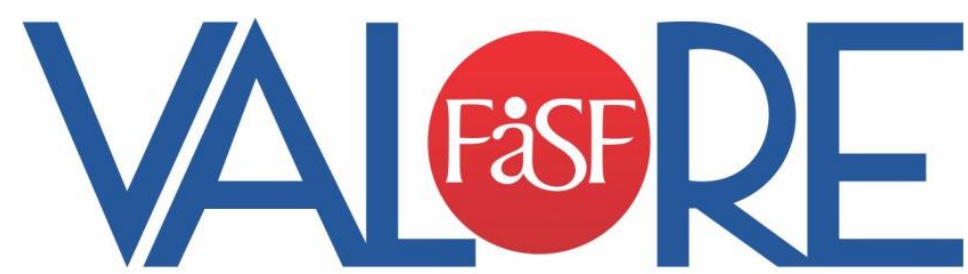

Revista Científica da FASF Faculdade Sul Fluminense

Volta Redonda, v.2 n.2 157p. jul./dez. 2017 ISSN 2525-9008 / e-ISSN 2526-043x

DOI: http://dx.doi.org/10.22408/reva112016 


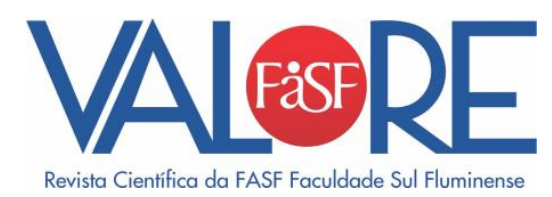

Revista Semestral Interdisciplinar das Coordenações de Cursos de Graduação e Pós-Graduação da FaSF

\title{
COMISSÃO EXECUTIVA
}

Editor Gerente: Prof. Me. Marcus Vinícius Barbosa, FaSF, Volta Redonda/RJ

\section{Editores}

Prof. Me. Adival de Sousa Monteiro, FaSF, Volta Redonda/RJ

Prof. Me. Felipe da Silva Triani, FGS, Rio de Janeiro/RJ

Profa. Me. Paloma de Lavor Lopes, FaSF/UGB, Volta Redonda/RJ

Profa. Me. Sônia Cardoso Moreira Garcia, UniFOA, Volta Redonda/RJ

\section{CONSELHO EDITORIAL}

Prof. Me. Alexandre Guandalini Bossa, IFAP/AP

Profa. Me. Aline Reis, FaSF/UGB, Volta Redonda/RJ

Profa. Dra. Ana Valéria Figueiredo da Costa, UERJ/UNESA Rio de Janeiro/RJ e UNIG, Nova Iguaçu/RJ Prof. Me. Antônio José de Figueiredo Pinto, UNIGRANRIO, Duque de Caxias/RJ

Prof. Dr. Carlos Alberto de Oliveira Magalhães, UEM. Maringá/PR

Prof. Me. Carlos Antônio Pereira da Silva, UNIGRANRIO, Duque de Caxias/RJ

Profa. Dra. Cristina Novikoff, UFCG, Cajazeiras/PB

Profa. Me. Débora Augusto Franco, FaSF/UGB, Volta Redonda/RJ

Prof. Me. Gabriel Luís da Conceição, UFRRJ, Seropédica/RJ, FaSF/UGB, Volta Redonda/RJ

Profa. Me. Hérica Cambraia Gomes, UBM, Barra Mansa/RJ

Profa. Me. Josycler Aparecida Arana, UFF - PUVR, Volta Redonda/RJ

Prof. Me. Marcos Porto Freitas da Rocha, UFRJ, Rio de Janeiro/RJ

Profa. Me. Patrícia Luciene da Costa Teixeira, UBM, Barra Mansa/RJ

Profa. Esp. Rafaela de Souza Santos, FaSF, Volta Redonda/ RJ

Profa.Me. Rhanica Evelise Toledo Coutinho, UniFOA, Volta Redonda/RJ

Capa ( criação e layout): Prof. Me. Álvaro Victório, Unigranrio, Duque de Caxias/RJ

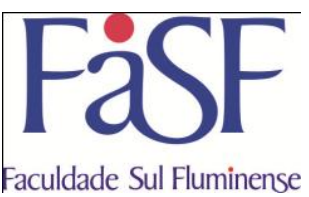

\author{
Mantenedora / Representante Legal \\ Prof. Lygia de Campos Telles \\ Corpo Diretivo \\ Diretor Geral \\ Prof. Claudio Alvares Menchise \\ Diretora Acadêmica \\ Prof $^{a}$ Denise Campos Telles Menchise \\ Vice Diretor \\ Prof. Dalton da Silva e Souza
}

Núcleo de Planejamento, Desenvolvimento e Pesquisa Institucional / Ouvidoria

Prof. Dalton da Silva e Souza 


\section{SUMÁRIO}

\section{EDITORIAL}

Prof. Dalton Silva e Souza

THE NEXUS BETWEEN ENERGY CONSUMPTION, ECONOMIC GROWTH, AND URBANIZATION IN LATIN AMERICAN AND CARIBBEAN COUNTRIES: AN APPROACH WITH PVAR MODEL

Matheus Koengkan

\section{O USO DAS TICs NO PROCESSO ENSINO E APRENDIZAGEM NA ESCOLA ALTERNATIVA “LAGO DOS CISNES”}

Maiara Capucho Costa, Maria Aparecida Silva de Souza

\section{VIOLËNCIA DE GËNERO: REFLEXÖES A PARTIR DO DOCUMENTÁRIO ESTAMIRA}

Rosane Cristina de Oliveira, Eliane Cristina Tenório Cavalcanti

PERSPECTIVAS HORIZONTAIS PARA O CURRICULO:C ONTRIBUIÇÕES TEÓRICAS E METODOLÓGICAS PARA A EDUCAÇÃO

Marcus Alexandre de Pádua Cavalcanti, Sandramor do Amaral Ferreira

IMAGENS DO INCONSCIENTE ALIANÇAS ENTRE ARTE ETERAPIA

Humberto Vieira, Marcus Alexandre de Pádua Cavalcanti, Eliane Cristina Tenório Cavalcanti

DICOTOMIA EXPERIËNCIA X COMPETËNCIA: O MERCADO DE TRABALHO PARA OS QUE PROCURAM O PRIMEIRO EMPREGO

Renan Gomes de Moura, Paloma de Lavor Lopes, Marcus Vinícius Barbosa

\section{DESAFIOS DA GESTÃO DE INSTITUIÇÕES DO TERCEIRO SETOR}

Leonardo de Paula Santoro, Marcus Vinícius Barbosa

\section{DESTILAÇÃO VIRTUAL DO VINHO COMO INSTRUMENTO REAL DE ENSINO APRENDIZAGEM DE QUÍMICA}

Eloísa Vieira, Denise Celeste Godoy, Hérica Cambraia Gomes

\section{ESTRATÉGIAS DE APRENDIZAGEM UTILIZADAS NO ENSINO DA NATAÇÃO PARAAUTISTAS}

Estefânia do Nascimento Soares, Victor Hugo do Rosario, Carlos Antônio Pereira da Silva, Felipe da Silva Triani

REDES DE INFRAESTRUTURA URBANA E SUAS RELAÇÕES COM OS COMPONENTES TÉCNICOS, SOCIAIS E AMBIENTAIS

Gunar Hernrique Guedes, Kairo Felipe dos Santos, Tânia do Carmo, Carlos Alberto de Oliveira Júnior

ESCOLA, CURRICULO E FORMAÇÃO DOCENTE: DESAFIOS NA CONTEMPORANEIDADE

Joelson Alves Onofre 


\section{EDITORIAL}

A Faculdade Sul Fluminense, passa por um momento bastante importante no cenário da Educação Superior da Região Sul Fluminense e do nosso Estado do Rio de Janeiro, não só com a conquista de novas unidades e ampliação de seus projetos educacionais, mas no resultado positivo obtido no ENADE e nas atividades sociais e culturais que vem promovendo.

Mais uma vez conquistamos o Selo de Instituição Socialmente responsável pela $A B M E S$ e a continuidade na construção da Revista Valore, com mais essa edição, que conta com estratos Qualis/CAPES "B4" em Ensino e "B5" em Ciências Ambientais, além de incrementar nossa base de dados e indexadores, reforçando nossa Missão de deixar a todos os discentes e docentes, boas lembranças de suas passagens pela vida acadêmica, bem como consolidar a posição para novos alcances de nosso periódico

Segue-se o momento de implementar atividades de educação continuada perpetuando a presença de todos em nosso convívio, bem como pela oportunidade de contarmos com um quadro valoroso de profissionais que com zelo acadêmico e envolvimento com a pesquisa, nos trazem grandes excelentes provocações acadêmicas.

Com alegria agradecemos à todos os colaboradores e parabenizamos por mais essa rica edição da Revista Valore.

Cordiais saudações acadêmicas,

Prof. Dalton Silva e Souza

Vice-Diretor

Faculdade Sul Fluminense 